\title{
Prognosis of Breast Cancer Patients Underwent Surgery in a Developing Country
}

\author{
Bangaly Traore ${ }^{1}$, Abdoulaye Toure ${ }^{2}$, Telly Sy ${ }^{3}$, Mamadou Moustapha Dieng ${ }^{4}$, \\ Mamoudou Condé1, Ahmadou Deme ${ }^{4}$, Namory Keita ${ }^{3}$ \\ ${ }^{1}$ Surgical Oncology Unit of Donka, University Hospital of Conakry, Conakry, Guinea \\ ${ }^{2}$ Department of Pharmacy, University of Conakry, Conakry, Guinea \\ ${ }^{3}$ Department of Gyneacology and Obstetric, Teaching Hospital of Conakry, Conakry, Guinea \\ ${ }^{4}$ Cancer Institute of Dakar, Dakar, Senegal \\ Email: ucodonka@gmail.com
}

Received 27 July 2015; accepted 28 August 2015; published 31 August 2015

Copyright (C) 2015 by authors and Scientific Research Publishing Inc.

This work is licensed under the Creative Commons Attribution International License (CC BY).

http://creativecommons.org/licenses/by/4.0/

(c) (i) Open Access

\begin{abstract}
Background: We aim to review different factors associated with the relapse and the mortality in breast cancer patients in restricted-resource settings over a five-year period. Method: A retrospective cohort study including 133 women underwent breast cancer surgery was performed at the Surgical Oncology Unit of the Conakry University Hospital in Guinea. Socio-demographical characteristics, clinical information, treatment and data on relapse and survival were retrieved from medical files. Predictor factors of relapse were analyzed by using univariate and multivariate logistic regression. Factors associated with mortality were analyzed by Kaplan Meier survival and Cox models. Results: Breast cancer surgery was conservative in 13 cases (9.8\%) and radical in 120 cases $(\mathbf{9 0 . 2 \% )}$. Five-year relapse was $33.8 \% \pm \mathbf{8 . 0} \%$ and the predictor factors of this relapse were age and stage III $(p=0.005)$. The overall 5 -year mortality was $42.1 \%$ and the risk factors independently associated with death were body mass index $(p=0.01)$, origin of patients $(p=0.02)$, radiotherapy $(p=0.01)$ and cancer relapse $(p=0.001)$. Conclusion: The proportions of relapse and death were higher in breast cancer patients. The age and the stage of cancer at the time of surgery were the predictor factors of relapse. Body mass index, origin of patients, radiotherapy and cancer relapse were associated with death.
\end{abstract}

\section{Keywords}

Breast Cancer, Breast Surgery, Prognosis, Developing Countries

\footnotetext{
"Corresponding author.
}

How to cite this paper: Traore, B., Toure, A., Sy, T., Dieng, M.M., Condé, M., Deme, A. and Keita, N. (2015) Prognosis of Breast Cancer Patients Underwent Surgery in a Developing Country. Journal of Cancer Therapy, 6, 803-810. 


\section{Introduction}

Cancer is recognized as a critical public health problem in Africa. While communicable diseases continue to burden African populations, it is becoming clear that non-communicable diseases also require the attention of health professionals.

Breast cancer is a major cause of morbidity and can reach high mortality among women. The incidence of this cancer has been progressively increasing worldwide. The number of new cases is estimated at 1.82 million in each year worldwide [1].

In Sub-Saharan Africa, the incidence of breast cancer ranged from 15 to 53 per 100,000 women, which are lower than those in industrialized countries [2]. However, the cancer burden reported for sub-Saharan Africa may be underestimated due to the lack or insufficient awareness, a lack of appropriate diagnosis, poor access to care, limitations in the technical workforce and infrastructure, and low quality of cancer data systems compared to those in high resource countries [3] [4]. In Guinea, the incidence of breast cancer is estimated at 14.5 new cases per 100,000 [5] and breast cancer accounts for over $26 \%$ of all cancers at our surgical oncology unit [6].

In developed countries, breast cancer is frequently diagnosed at an early stage allowing conservative treatment and reducing mortality due to this disease. In countries with limited resources, locally advanced breast cancer is still common and has poor prognosis [2] [7] [8]. In sub-Saharan Africa, data on survival of breast cancer patients are limited [9].

Recent advances in the diagnosis (molecular subtypes) and treatment (targeted therapy), allowing to individualize breast cancer treatment, are still poorly available in developing countries [10].

In the present study, we aimed to analyze the predictive factors of relapse and other events that are associated with five-year survival after breast cancer surgery in our restricted-resource settings.

\section{Materiel and Methods}

\subsection{Setting}

The study was conducted in the surgical oncology unit of the University Hospital of Conakry, Guinea.

\subsection{Study Design and Population}

This was a retrospective cohort study on 133 patients with histologically confirmed breast cancer from 2005 to 2012. These patients underwent surgical operation and were followed from November 2005 to February 2014.

\subsection{Data Collection}

For each patient, data were collected from the date of cancer diagnosis until the end of the follow up, i.e. 60 months after surgery. Data included socio-demographical information (age at cancer diagnosis, marital status, profession, residence area) and anthropometric characteristics (body mass index) source of referral (home, hospital), co-morbidities, cancer characteristics (date of diagnosis, pathological statute, molecular subtypes, location of primary cancer, stage), date and type of surgery, other cancer treatment (radiotherapy, chemotherapy). For cancers diagnosed before 2010, the stage at diagnosis was revised according to the TNM classification of the UICC 2010 [10] at the early (stage I-II) and late stage (stage III).

\subsection{Surgical Treatment Procedures}

Surgical treatment was dependent on the stage at diagnosis. For the breast cancer in the stage I, breast-conserving surgery (BCS) was performed. In stage II, neoadjuvant chemotherapy (NAC) was administrated before BCS or radical breast surgery (RBS). In stage III, RBS was performed after NAC. The RBS applied was a modified Patey's method. NAC was based on a protocol comprising anthracyclines and/or taxanes. After surgery, adjuvant chemotherapy was administered. There is no radiotherapy in our country and patients are referred to the Institute of Cancer Dakar (Senegal) for this. Adjuvant hormonal therapy (tamoxifen) was also prescribed for menopausal women and/or with positive hormone receptors.

\subsection{Statistical Analysis}

Patients were stratified into two groups: patients experiencing relapse and patients without relapse. Categorical 
variables were shown as the frequency and percentage (\%), and continuous variables were presented as the median and interquartile range (IQR). Frequency distributions between categorical variables were compared using $\chi^{2}$ test or Fisher's exact test while continuous variables were compared using the Mann-Whitney test. A multivariable Cox proportional hazards model was used to estimate the Hazard Ratio (HR) and its corresponding 95\% confidence interval (CI). Survival distributions were analyzed using the Kaplan-Meier method. For all tests performed, 2-tailed $P$ values $<0.05$ were regarded as denoting statistical significance. Statistical data were analyzed with statistical package for the social sciences (version 21.0 for Windows, SPSS, Inc., Chicago, IL).

\subsection{Ethical Considerations}

In this retrospective study, data were collected anonymously and confidentially. Patients signed the consent form for the use of data contained in their records.

\section{Results}

\subsection{Characteristics of Patients}

A total of 133 consecutive patients who underwent surgery for a primary breast cancer were included in this study. The median age of patients was $48.0 \pm 12.4$ years. Table 1 shows that the patients were mostly the housewives (51.8\%) and vendors (9.1\%), and about half of them live in Conakry (53.6\%), the capital of the country.

Cancer characteristics showed that the main histological type was invasive ductal carcinoma (86 cases or 64.7\%), 27 cases of carcinoma no other specified (20.3\%), 10 cases of invasive lobular carcinoma (7.5\%) and 4 cases of mucinous carcinoma (3.0\%).

In 24 cases, according to the hormone receptor status and the Her2 oncogene, the molecular subtypes were luminal A (29.2\%), luminal B (8.3\%), triple negative (29.2\%) and Her 2 positive (33.3\%).

Breast cancer surgery was conservative in 13 cases (9.8\%) and radical in 120 cases (90.2\%). The median time from diagnostic to surgery was 5.0 months (IQR: 3.0 - 7.0). In addition to surgery, $90 \%$ of all patients received a chemotherapy and $44.4 \%$ a radiotherapy.

Table 1. Socio-demographical characteristics of breast cancer patients who had a surgical management.

\begin{tabular}{cccc}
\hline Characteristics & Relapse $\mathbf{n}=\mathbf{4 5}$ & Without relapse $\mathbf{n}=\mathbf{8 8}$ & $\mathbf{P}$ \\
\hline Age, years & $43.4 \pm 9.8$ & $50.5 \pm 13.0$ & 0.001 \\
Body mass index & $26.9 \pm 6.0$ & $25.6 \pm 4.8$ & 0.37 \\
Menopause & $14(31.8)$ & $52(59.8)$ & 0.003 \\
Profession & & $10(12.3)$ & 0.21 \\
Vendors & $6(15.4)$ & $47(58.0)$ \\
House wives & $17(43.6)$ & $6(7.4)$ \\
Dressmakers & $3(7.7)$ & $3(3.7)$ & $3(3.7)$ \\
Teachers & $6(15.4)$ & $3(3.7)$ \\
Health workers & $3(7.7)$ & $9(11.1)$ \\
Engineers & $0(0.0)$ & & 0.38 \\
Secretaries/administrators & $4(10.3)$ & $52(59.1)$ \\
Geographical areas & & $36(40.9)$ \\
Conakry and countryside & $23(51.1)$ & \\
Other country's areas & $22(48.9)$ & $6(28.6)$ \\
Underlies diseases & $2(28.6)$ & $20(22.7)$ \\
Diabetes mellitus & $11(24.4)$ & & 1.00 \\
Arterial hypertension & & & 0.82 \\
\hline
\end{tabular}




\subsection{Predictive Factors of Cancer Relapse}

Out of all patients, 45 experienced relapse, corresponding to a 60 -month relapse rate of $33.8 \% \pm 8.0 \%$. Relapse occurred in the first year after surgery in 9 cases $(20.0 \%)$, the second year in 18 cases $(40.0 \%)$, the third year in 12 (26.7\%), the fourth year in 0 cases (0.0\%), and the fifth year in 6 cases (13.3\%). Of the all of these relapses, 2 (4.4\%) were local, 4 (8.9\%) were loco-regional, 19 (42.2\%) were metastatic and 11 (24.4\%) were in more sites. The median time from surgery to relapse was 7.0 months (IQR: 3.0 - 15).

Socio-demographic and cancer characteristics in patients who relapsed and those who did not relapse are presented in Table 1 and Table 2 respectively. The median time from diagnosis to surgery was 6 (IQR: 3.5 - 8.5) and 4 months (IQR: $3.0-6.0)$, respectively in the relapse and no relapse patients $(P=0.04)$. It is worth noting that at the time of diagnosis, the primary cancer was more advanced in patients who experienced relapse. In these patients, cancer was mainly at stage III $(P=0.03)$. The results of logistic multivariate regression showed that the predictor factors of relapse were age [OR $=0.94 ; 95 \% \mathrm{CI}(0.92-0.99 ; P=0.005)]$, and the stages III and IV of cancer $[\mathrm{OR}=6.30 ; 95 \% \mathrm{CI}(1.72-22.97) ; P=0.005)]$. The body mass index was retained in the model but the association was not statistically significant (OR $=1.10 ; 95 \% \mathrm{CI}(0.99-1.22 ; P=0.08)$.

\subsection{Five-Year Mortality}

Out of 133 patients, 56 died corresponding to an overall 60-month mortality of 42.1\% (95\%CI: 33.6\% - 50.6\%). Mortality was higher in patients with relapse than in patients with no relapse $(64.3 \%$ vs. $35.7 \%$; $P<0.001)$. The median time from diagnosis to death was 18.0 (IQR: 11.0 - 29.0) months. The results of the multivariate Cox model showed that the independently factors associated with death were body mass index, origin of patients, radiotherapy and cancer relapse (Table 3).

\section{Discussion}

Breast cancer is a major cause of morbidity and cancer related mortality among African women. In the current study, we analyzed the factors associated with the occurrence of relapse and mortality of breast cancer patients who underwent surgery in our surgical oncology unit in Conakry, Guinea. Of 276 women with breast cancer followed in that unit, only $50.7 \%$ underwent surgery. This could explain the low availability of cancer treatment facilities in low-resource countries. Limited financial resources lead to a suboptimal cancer data collection, as well as a delayed diagnosis and treatment, a common characteristic of many African breast cancer patients [9] [11].

The results showed that breast cancer relapse is the most common. The five-year relapse rate was 33.8\%. This high relapse rate could be explained by the young age of the patients, premenopausal status, advanced stage

Table 2. Comparison of the breast cancer characteristics in patients with relapse and without relapse after surgery.

\begin{tabular}{cccc}
\hline & Relapse $\mathbf{n}=\mathbf{4 5}$ & Without relapse $\mathbf{n}=\mathbf{8 8}$ & $\boldsymbol{P}$ \\
\hline Cancer stage & & & 0.002 \\
Stage I - II & $3(6.7)$ & $27(30.7)$ & \\
Stage III - IV & $42(93.3)$ & $61(69.3)$ & \\
Surgical treatment & & & 0.004 \\
Conservative surgery & $0(0.0)$ & $13(14.8)$ & \\
Radical mastectomy & $45(100.0)$ & $75(85.2)$ & \\
Chemotherapy & $41(91.1)$ & $80(92.7)$ & 0.00 \\
Radiotherapy & $17(37.8)$ & $42(47.7)$ & 0.77 \\
Origin of patients & & & \\
Hospital & $28(62.2)$ & $57(64.8)$ & \\
Home & $17(37.8)$ & $31(35.2)$ & \\
\hline
\end{tabular}


Table 3. Risk factors independently associated with mortality in breast cancer patients underwent surgery at Donka National Hospital.

\begin{tabular}{cccc}
\hline & Adjusted HR & 95\% CI & P \\
BMI & 1.10 & $1.02-1.18$ & 0.01 \\
Origin of patients & & & 0.02 \\
Home & Ref & $1.12-4.09$ & 0.01 \\
Hospital & 2.14 & $0.25-0.88$ & 0.001 \\
Radiotherapy & 0.46 & $3.65-13.42$ & \\
Relapse & 7.00 & & \\
\hline
\end{tabular}

cancer and radical surgery. Patients who relapsed were younger than patients who did not relapse $(P=0.001)$. Similar data have been reported in previous studies previous data [12]-[14].

The median time from surgery to relapse was 7 months, and over $80 \%$ occurred in 3 years after surgery. This is consistent with data of previous studies in the developing countries in which the relapse rate within 5 years was around of $80 \%$ [15] [16]. In Finland, the authors found that the median time from surgery to relapse was 27 months [17]. These data suggest that relapse is earlier in low income countries than industrialized countries.

Data showed that in $77.4 \%$ of all patients, cancer was at stage III at the time of diagnosis. This suggests that the diagnosis was delayed and this contributed to an increase of the rate of relapse. In fact, 93.3\% who experienced a cancer relapse were at stage III.

During the study period, no relapse was noted in patients who underwent breast conserving surgery. Conversely, all cases of relapse occurred in patients with radical breast surgery. Data from several studies showed that there are differences in the risk of relapse and survival but not statistically significant between breast-conserving surgery and radical breast surgery [18] [19]. In a Brazilian study, the recurrence rate after breast-conserving surgery was $6.8 \%$ [20].

In this study, almost all of the patients received the chemotherapy. However, the high rate of locoregional and metastatic relapse was higher. This could be explained by the aggressive behavior of tumor.

In our patients, data showed that radiotherapy improves the outcome of locoregional control and reduces the risk of relapse after surgery. Thus, relapse-free survival of locally-advanced breast cancer can reach $87 \%$ in patients treated with adjuvant radiotherapy [21]. This suggests that the availability of radiotherapy in the country would be helpful for improving breast cancer survival in developing countries.

The 5-year overall mortality was $42.1 \%$ and this was higher in patients who experienced relapse. Previous data showed that the 5-year mortality was from 36\% to 38\% in Sub-Saharan Africa [22]-[24]. The overall survival and disease-free survival $57.9 \%$ and $64.3 \%$ respectively are similar to other previous studies performed in the developing countries [25]-[29] (Table 4).

The multivariate Cox models showed that the increasing of BMI was an independently factor associated with death. In a meta-analysis, Chan et al. [30], showed the associations of obesity with an unfavorable overall and breast cancer survival in pre and post-menopausal breast cancer, regardless of when body mass index is ascertained. These findings suggest that as in developed countries, obesity is a condition to be taken into account in the fight against non-communicable diseases in developing countries as well. Thus regular physical exercise shown to have positive effects in the prevention of breast cancer and in improving the daily life of breast cancer patients [31] must be promoted among African women.

We noted that patients who came from other services of the hospital were at a high risk to death. We believe that patients lose enough time going through the non-specialist services where diagnostic facilities are very limited before reaching an oncology service. In a recent study, we reported that despite the majority of breast cancers are referred to our unit for healthcare professionals, the diagnosis is still late [32]. This could be related to the multiple recourses used by patients before the consultation in specialized oncology services. Gombe Mbalawa et al. [33] showed that the late diagnosis of breast cancer was largely attributed to the responsibility of health professionals: the hospital personal, the nurses and physicians who work in private $(40.8 \%)$ and the medical doctor, specialist or not (25.5\%) while the direct responsibility of the patient was weak (24.4\%) by ignorance or fear of diagnosis. 
Table 4. Comparison of overall survival, disease-free survival and independent prognostic factors for 5-year follow-up of breast cancer in some developing countries.

\begin{tabular}{cccccc}
\hline Studies & Number of cases & Country & OS & DFS & Independent prognosis factors \\
\hline Our study & 133 & Guinea & $57.9 \%$ & $64.3 \%$ & BMI, Origin of patients, radiotherapy, relapse \\
Al-Moundhri et al. 2004 & 152 & Oman & $62 \%$ & $64 \%$ & $\begin{array}{c}\text { Axillary node involvement } \\
\text { Tumor differentiation }\end{array}$ \\
Ben Gobrane et al. 2007 & 470 & Tunisia & $61 \%$ & NA & $\begin{array}{c}\text { Surgery, radiotherapy } \\
\text { Gakwaya } \text { et al. } 2008\end{array}$ \\
$\begin{array}{c}\text { Makanjuola } \text { et al. } 2014 \\
\text { Ngowa } \text { et al. } 2015\end{array}$ & 224 & Uganda & $56 \%$ & NA & Stage \\
\hline
\end{tabular}

NA: Not available.

Despite the importance of this study and its potential impact on the quality of care for patients with breast cancer in developing countries, it may have some limitations: first, because of the design which was a retrospective cohort study. Somme important data as molecular subtypes were missed in the majority of patients because this test is not carried in our country. Secondly, we cannot be taking into account of socio-economical (education, income) data, because they were not recorded. However, in Guinea, the medical care is not free and there is no almost any health insurance. So, patients pay medical care. Thus, the taken into account of socioeconomical data in the regression would be helpful to the best understand of the determinants of the prognosis after breast cancer surgery. Finally, the sample size was small. Need to be extended in a large study with a high number of samples. However, despite the small size, the power of this retrospective study can be considered as sufficient.

\section{Conclusion}

The proportions of relapse and death were higher in breast cancer patients. Age and stage of cancer at time of surgery were the predictors of relapse and body mass index, radiotherapy and cancer relapse were associated with death. The improved means of diagnosis and access to other methods of locoregional and systemic treatment provide a better understanding and increase the survival of breast cancer treated in our settings. The findings of this study will be helpful for the physicians in developing countries in better referral of the patients from the cancer management health department. The impact of the study is to provide the first breast cancer survival data in a country with low resources.

\section{Acknowledgements}

We thank Dr Bakary Sidiki Sylla for his advice and for the critical review of the manuscript.

\section{Conflict Interests}

No conflict interests.

\section{References}

[1] Ferlay, J., Soerjomataram, I., Dikshit, R., et al. (2014) Cancer Incidence and Mortality Worldwide: Sources, Methods and Major Patterns in GLOBOCAN 2012. International Journal of Cancer, 136, E359-E386.

[2] Ly, M., Antoine, M., André, F., et al. (2011) Breast Cancer in Sub-Saharan African Women: Review. Bulletin du Cancer, 98, 797-806.

[3] Amadori, D., Serra, P., Bravaccini, S., et al. (2014) Differences in Biological Features of Breast Cancer between Caucasian (Italian) and African (Tanzanian) Populations. Breast Cancer Research and Treatment, 145, 177-183. http://dx.doi.org/10.1007/s10549-014-2903-0

[4] Morhason-Bello, I., Odedina, F., Rebbeck, T.R., et al. (2013) Challenges and Opportunities in Cancer Control in Africa: A Perspective from the African Organisation for Research and Training in Cancer. The Lancet Oncology, 14, e142e151. http://dx.doi.org/10.1016/s1470-2045(12)70482-5

[5] Globocan 2012. (2014) Estmated Cancer Incidence, Mortality and Prevalence Worldwide. http://globocan.iarc.fr 
[6] Traore, B., Barry Sonna, M., Dassy, E., et al. (2011) Over Review of Three Years (2007-2009) Activities of the Unit of Surgical Oncology, National Hospital of Donka. Guinée Médicale, 72, 11-18.

[7] Dickens, C., Joffe, M., Jacobson, J., et al. (2014) Stage at Breast Cancer Diagnosis and Distance from Diagnostic Hospital in a Periurban Setting: A South African Public Hospital Case Series of over 1000 Women. International Journal of Cancer, 135, 2173-2182. http://dx.doi.org/10.1002/ijc.28861

[8] Abdulrahman Jr., G.O. and Rahman, G.A. (2012) Epidemiology of Breast Cancer in Europe and Africa. Journal of Cancer Epidemiology, 2012, Article ID: 915610. http://dx.doi.org/10.1155/2012/915610

[9] Sankaranarayanan, R., Swaminathan, R., Brenner, H., et al. (2010) Cancer Survival in Africa, Asia, and Central America: A Population-Based Study. The Lancet Oncology, 11, 165-173. http://dx.doi.org/10.1016/S1470-2045(09)70335-3

[10] Galukande, M., Wabinga, H. and Mirembe, F. (2015) Breast Cancer Survival Experiences at a Tertiary Hospital in Sub-Saharan Africa: A Cohort Study. World Journal of Surgical Oncology, 13, 220. http://dx.doi.org/10.1186/s12957-015-0632-4

[11] Sobin, L.H., Gospodarowicz, M.K. and Wittekind, C. (2009) The TNM Classification of Malignant Tumors. 7th Edition, UICC.

[12] Fregene, A. and Newman, L.A. (2005) Breast Cancer in Sub-Saharan Africa: How Does It Relate to Breast Cancer in African-American Women? Cancer, 103, 1540-1550. http://dx.doi.org/10.1002/cncr.20978

[13] López, M.A., Latiesas, X.S., Arnalot, P.F., Bassols, M.L., Castillejo, A.R., Jordana, J.Q., et al. (2007) Risk Factors of Local Relapse in Breast Cancer: The Importance of Age. Clinical and Translational Oncology, 9, 110-116. http://dx.doi.org/10.1007/s12094-007-0021-8

[14] Wei, X.Q., Li, X., Xin, X.J., Tong, Z.-S. and Zhang, S. (2013) Clinical Features and Survival Analysis of Very Young (Age < 35) Breast Cancer Patients. Asian Pacific Journal of Cancer Prevention, 14, 5949-5952. http://dx.doi.org/10.7314/APJCP.2013.14.10.5949

[15] Chauleur, C., Vulliez, L., Trombert, B., Raoux, D., Khaddage, A. and Seffert, P. (2008) Risk Factors for Tumor Recurrence after Breast Conserving Therapy: About 254 Cases. Journal de Gynécologie Obstétrique et Biologie de la Reproduction, 37, 170-178. http://dx.doi.org/10.1016/j.jgyn.2007.11.027

[16] Yu, K.D., Li, S. and Shao, Z.M. (2011) Different Annual Pattern Lumpectomy and Mastectomy: Implication for Breast Cancer Surveillance after Breast-Conserving Surgery. Oncologist, 16, 1101-1110. http://dx.doi.org/10.1634/theoncologist.2010-0366

[17] Apffelstaedt, J.P. (2003) Locally Advanced Breast Cancer in Developing Countries: The Place of Surgery. World Journal of Surgery, 27, 917-920. http://dx.doi.org/10.1007/s00268-003-6974-z

[18] Siponen, E.T., Joensuu, H. and Leidenius, M.H. (2013) Local Recurrence of Breast Cancer after Mastectomy and Modern Multidisciplinary Treatment. Acta Oncologica, 52, 66-72. http://dx.doi.org/10.3109/0284186X.2012.718793

[19] Freedman, G.M. and Fowble Barbara, L. (2000) Local Recurrence after Mastectomy or Breast-Conserving Surgery and Radiation. Oncology, 14, 1561-1581.

[20] Cho, J.H., Park, J.M., Park, H.S., Park, S., Kim, S.I. and Park, B.-W. (2013) Oncologic Safety of Breast-Conserving Surgery Compared to Mastectomy in Patients Receiving Neoadjuvant Chemotherapy for Locally Advanced Breast Cancer. Journal of Surgical Oncology, 108, 531-536. http://dx.doi.org/10.1002/jso.23439

[21] Tovar, J.R., Zandonade, E. and Amorim, M.H. (2014) Factors Associated with the Incidence of Local Recurrences of Breast Cancer in Women Who Underwent Conservative Surgery. International Journal of Breast Cancer, 2014, Article ID: 639534. http://dx.doi.org/10.1155/2014/639534

[22] But-Hadzić, J., Bilban-Jakopin, C. and Hadzić, V. (2010) The Role of Radiation Therapy in Locally Advanced Breast Cancer. The Breast Journal, 16, 183-188. http://dx.doi.org/10.1111/j.1524-4741.2009.00885.x

[23] Wabinga, H., Parkin, D.M., Nambooze, S. and Amero, J. (2011) Cancer Survival in Kampala, Uganda, $1993-1997$. IARC Scientific Publications, 162, 243-247.

[24] Al-Moundhri, M., Al-Bahrani, B., Pervez, I., Ganguly, S.S., Nirmala, V., Al-Madhani, A., et al. (2004) The Outcome of Treatment of Breast Cancer in a Developing Country-Oman. Breast, 13, 139-145. http://dx.doi.org/10.1016/j.breast.2003.10.001

[25] Kantelhardt, E.J., Zerche, P., Mathewos, A., Trocchi, P., Addissie, A., Aynalem, A., et al. (2014) Breast Cancer Survival in Ethiopia: A Cohort Study of 1,070 Women. International Journal of Cancer, 135, 702-709. http://dx.doi.org/10.1002/ijc.28691

[26] Ben Gobrane, H., Fakhfakh, R., Rahal, K., Ben Ayed, F., Mâalej, M., Ben Abdallah, M., et al. (2007) Breast Cancer Prognosis in Salah Azaiez Institute of Cancer, Tunis. Eastern Mediterranean Health Journal, 13, 309-318.

[27] Gakwaya, A., Kigula-Mugambe, J.B., Kavuma, A., Luwaga, A., Fualal, J., Jombwe, J., et al. (2008) Cancer of the Breast: 5-Year Survival in a Tertiary Hospital in Uganda. British Journal of Cancer, 99, 63-67. 
http://dx.doi.org/10.1038/sj.bjc.6604435

[28] Makanjuola, S.B., Popoola, A.O. and Oludara, M.A. (2014) Radiation Therapy: A Major Factor in the Five-Year Survival Analysis of Women with Breast Cancer in Lagos, Nigeria. Radiotherapy and Oncology, 111, 321-326. http://dx.doi.org/10.1016/j.radonc.2014.03.013

[29] Ngowa, J.K.D., Kasia, J.M., Yomi J., Nana, A.N., Ngassam, A., Domkam, I., et al. (2015) Breast Cancer Survival in Cameroon: Analysis of a Cohort of 404 Patients at the Yaoundé General Hospital. Advances in Breast Cancer Research, 4, 44-52. http://dx.doi.org/10.4236/abcr.2015.42005

[30] Chan, D.S., Vieira, A.R., Aune, D., Bandera, E.V., Greenwood, D.C., McTiernan, A., et al. (2014) Body Mass Index and Survival in Women with Breast Cancer-Systematic Literature Review and Meta-Analysis of 82 Follow-Up Studies. Annals of Oncology, 25, 1901-1914. http://dx.doi.org/10.1093/annonc/mdu042

[31] Ogunleye, A.A. and Holmes, M.D. (2009) Physical Activity and Breast Cancer Survival. Breast Cancer Research, 11, 106. http://dx.doi.org/10.1186/bcr2351

[32] Traoré, B., Keita, M., Diane, S., Dankoro, A., Kabba, I.S. and Keita, N. (2012) Clinicopathological Study of Breast Diseases Presenting to the Surgical Oncology Unit of Donka University Hospital in Conakry, Guinea. West African Journal of Medicine, 31, 227-231.

[33] Gombé Mbalawa, C., Diouf, D., NkouaMbon, J.B., Minga, B., Makouanzi Nsimba, S. and Nsondé Malanda, J. (2013) Arrival of Patients at Advanced Stage: Tempting to Identify Responsibility. Bulletin du Cancer, 100,167-172. 